\title{
Empirical Analysis of the Spatial Genetic Algorithm on Small-World Networks ${ }^{\star}$
}

\author{
Yong Min ${ }^{1}$, Xiaogang $\mathrm{Jin}^{1,2, \star \star}$, Xianchuang $\mathrm{Su}^{3}$, and Bo Peng ${ }^{1}$ \\ 1 AI Institute, College of Computer Science, Zhejiang university, \\ Hangzhou 310027, China \\ 2 Ningbo Institute of Technology, Zhejiang university, Ningbo 315100, China \\ xiaogangj@cise.zju.edu.cn \\ 3 College of Software Engineering, Zhejiang university, Hangzhou 310027, China
}

\begin{abstract}
Genetic algorithm (GA) has been widely used in optimizing and solving various problems since first proposed, and its characters also have been deeply studied. In this paper, we investigate the benefits of genetic algorithm whose population is distributed on small-world networks. In particular, we pay our attention to the complexity of how small-world affects the behavior of spatial GA. Our work shows that, on a complex problem, the behavior of spatial GA on the small-world networks is influenced by at least two different factors: local selection and asymmetric topology. It is more complex than previous results from simple lattice models. Our results could provide lots of potential methods to improve the performance of spatial GA and give some guidance for designing of parallel genetic algorithm. We also present many future problems on the influence of small-world to spatial GA.
\end{abstract}

\section{Background}

\subsection{Spatial GA and Local Selection Effect}

Influence of spatial structure on evolution has been researched from various fields: biology [1] 8] [10, mathematics [6] and computer science 9] 11]. These researches have proved that the spatial structure would deeply affect evolution. Spatially structured population has been also proposed in genetic algorithm as a tool for improving the searching properties of GA. Spatial GA is a kind of genetic algorithm whose population has certain spatial structure. The previous studies on spatial structure of population were primarily related to the parallelism genetic algorithm (PGA) [3] [5. Here, we should mention about fine grained parallel genetic algorithm: the population is distributed on a network, therefore, one individual and his directly connected neighbors form a deme. Any individual could only interact with their neighbors, when we perform operators of GA, such as selection or mutation. The authors noted that the performance of the algorithm

\footnotetext{
* Supported by Zhejiang Provincial Natural Science Foundation of China under Grant No. Y105697 and Ningbo Natural Science Foundation (2005A610004).

** Corresponding author.
} 
degraded as the size of the deme increased. This effect, called local selection, has been researched further by Jayshree Sarma and Kenneth De Jong[7]. Their results show that the critical parameter of spatial GA is the ratio of the radius of the neighborhood to the radius of the underlying networks. Moreover, the coefficient of the growth rate of the best individual in the population is shown to be an inverse exponential function of this ratio.

\section{$1.2 \quad$ Small-World}

The networks that occur in lots of social, biological, and man-made systems are usually neither completely regular (such as lattices), nor completely random. These networks usually possess two characters: one is average vertex-to-vertex distances increase only logarithmically with the total number of vertices, another is so-called "clustering" that two neighbors of a vertex will often also be the neighbors of another. The networks that have above two characters are named "Small-World". In order to study the spatial GA on the small-world networks, we need a artificial model of small-world networks. Watts and Strogatz [2] have proposed an famous model for the small-world (WS model), which perhaps fits better than real small-world networks. Fig.1.1 and Fig.1.2 schematically describes the process for building small-world of WS model. The details of WS mode can be found at [2].

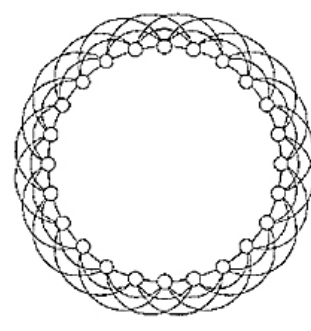

(1)

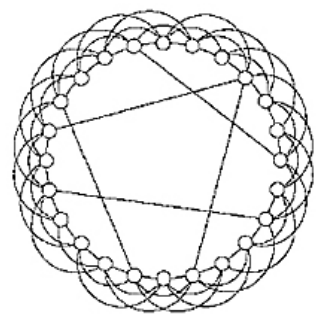

(2)

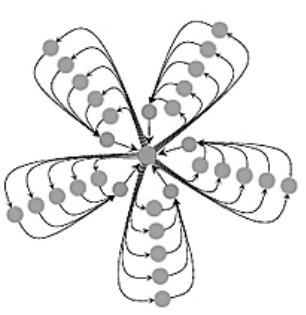

(3)

Fig. 1. The Watts-Strogatz Model. AND Super-Star Structure.

1) The regular lattice with periodic boundary conditions, so that the system becomes a ring. Each vertex connected to its $z$ nearest neighbors, where in this case $z=6$ (radius of the neighorhood $r_{n}: 2 r_{n}=z$ ). 2) The Watts-Strogatz model is created by rewiring a small fraction of the links (in this case five of them) to new sites chosen at random.

3) The super-star structure.

\subsection{Asymmetric Topological Structure}

More recent discoveries of spatial influence of evolution, from Erez Lieberman et al. 4, suggest that the amplification of selection occurs at a large amount of vertices feeds into single vertices which then feed into possibly further single vertices until they feed into the central hub and finally the hub feeds back into the these vertices. This feedback loop acts as a filter that reduces stochastic effect of 
random drift. Fig.1.3 illustrates a such structure 4]. Generally, in a network, we define that the sum of weight of any vertex's leaving links $\left(W_{\text {out }}\right)$ is 1 . Therefore, for a vertex, if sum of weight of entering links $\left(W_{i n}\right)$ is equal to 1 , this vertex neither suppress nor amplify selection; if $W_{\text {in }}<1$, this vertex amplify selection; if $W_{i n}>1$, this vertex suppress selection. In sum, the asymmetric topology could affect the behaviors of evolution.

In this paper, it is the first time that explores the effects of asymmetric topology for genetic algorithm. We also want to illustrate that the behavior of spatial GA on small-world is complicate, which is affected by at least two factors: local selection and asymmetric topology.

\section{Experimental Setup}

\subsection{Algorithm}

Our experiments base on Matlab's Genetic Algorithm and Direct Search (GADS) toolbox, and we modify its algorithm to fit for our requirements. The more details of Matlab's GADS toolbox can be found in Matlab's documents and website 12 .

In our GA (we call it NGA: genetic algorithm on networks) which is similar with grained parallel GA, but it run on a single machine, the population is distributed on small-world networks generated by WS model. NGA has three main steps which are differ from standard genetic algorithm(SGA: Standard algorithm in Matlab): First step is preserving several elites to next generation according to the ranking of individuals' fitness. One individual just preserve one time. The preserved individuals will be stored in the same position at network as their current position. Second step is generating mutation offspring whose number is $m$. In the step, we first choose $m$ unoccupied vertices from underlying network, which are labelled by $v_{i},(i=1,2, \ldots, m)$. For each $v_{i}$, we should choose a parent from the individuals in $v_{i}$ and its neighbors, and the mutation of the parent will occupy $v_{i}$ in next generation. Third step is generating crossover offspring. Generating method likes method described in second step, but it needs two parents for one offspring and the offspring is generated by crossover operator. The main difference between NGA and SGA is whether the sequence or position of individuals can be ignored. SGA's population is a simple set of individuals, but structure of population of NGA is corresponding to the underlying network structure.

\subsection{Definition of Success and the Diversity of Population}

At the end of algorithm running, the best individual's fitness is $f_{e}$, and the target fitness is $f_{t}$. In our experiments, the value of fitness is just the value of target functions. We say running of algorithm is success, if $\left|f_{e}-f_{t}\right|<0.01$.

In our NGA, the coding space is $\{0,1\}^{L}$, size of population is $n$. The population is $P=\left\{a_{1}, a_{2}, \ldots, a_{n}\right\}$, where $a_{j}=\left(a_{1 j}, a_{2 j}, \ldots, a_{L j}\right), j=1,2, \ldots, n$, so, the diversity of population is defined as:

$$
D(P)=1-\frac{1}{L \cdot n} \sum_{l=1}^{L}\left(\max \left\{\sum_{j=1}^{n}\left(a_{l j}\right), \sum_{j=1}^{n}\left(1-a_{l j}\right)\right\}-\min \left\{\sum_{j=1}^{n}\left(a_{l j}\right), \sum_{j=1}^{n}\left(1-a_{l j}\right)\right\}\right) ;
$$


obviously, $0 \leq D(P) \leq 1$. When $D(P)=0$, the diversity of population is minimal, and it is maximal, when $D(P)=1$.

\subsection{Experiment 1}

In order to ensure that the results of our experiments are correct, we need to prove NGA is "equivalent to" SGA, when NGA plays on a fully connected network. It is imply that the improving or worsening comes from small-world effect but from program. Here, we use Rastrigin's function, which is commonly used for testing genetic algorithm, to test both NGA on fully connected networks and SGA. The definition of two-dimensional Rastrigin's function is: $R_{1}(x, y)=$ $10 \cdot n+\left[x^{2}-10 \cdot \cos (2 \pi x)\right]+\left[y^{2}-10 \cdot \cos (2 \pi y)\right]$, and the target task is: $\min \left(R_{1}\right)$, where $x, y \in[-5.12,5.12]$.

The NGA has been defined above, and SGA come from the Matlab toolbox. Main parameters for Matlab's GA algorithm are presented in below table:

\begin{tabular}{|l|l|}
\hline Parameter Name & Value \\
\hline \hline Population Type & bitstring (length is 18) \\
\hline Elite Count & 10 percents of population \\
\hline Crossover Probability & $80 \%$ \\
\hline Maximal Generation & 100 \\
\hline Selection Method & roulette \\
\hline Crossover Method & single point \\
\hline
\end{tabular}

what is meaning of these parameters and how they affect the behavior of algorithm can be found in Matlab documents 12. Other default parameters also can be found in the documents. Every algorithm will repeat 100 times independently, and we will compare NGA with SGA at these parameters: success rate, diversity of population and convergent speed. From those, we want to prove that NGA is "equivalent to" SGA.

\subsection{Experiment 2}

In this experiment, we want to explore how small-world affects the performance and behavior of spatial GA. Therefore, we will execute NGA on a series of small-world networks of WS model. These small-world networks base on onedimensional lattice that have 256 vertices and $r_{n}=2$. The rewiring probability $p_{i},(1 \leq i \leq 29)$ of those networks is:

$$
p_{i}= \begin{cases}(i-1) / 256 & ; \quad 1 \leq i \leq 21 \\ (i-21) / 10 & ; \quad 22 \leq i \leq 29\end{cases}
$$

Therefore, there are 29 different networks: $G_{i}$. $G_{i}$ 's rewiring probability is $p_{i}$, where $1 \leq i \leq 29$.

We choose Rosenbrock's function: $R_{2}(x, y)=100 \cdot\left(y-x^{2}\right)^{2}+(x-1)^{2}$ for fitness function, and target task is: $\min \left(R_{2}\right)$, where $x, y \in[-5.12,5.12]$. (SGA 
performs badly in Rosenbrock's function. Therefore, it fits for describing the improvement and the properties of NGA on small-world.) The parameters of algorithm in experiment 1 and 2 are identical except the maximal generation is 200 in this experiment and every algorithm will repeat 120 times independently.

In regular lattices, the degree of any vertex is identical. It is 4 in this experiment. By rewiring, many vertices' degree would be bigger than 4, and some others would be smaller than 4 . Here, vertices are labeled by $v_{i}, 1 \leq i \leq 256$, and the degree of vertices is labeled by $\operatorname{degree}\left(v_{i}\right)$. we define asymmetric factor $U$ as:

$$
U=\sum \text { degree }\left(v_{i}\right), \text { where degree }\left(v_{i}\right)>4,1 \leq i \leq 256
$$

Although this is a crude definition for asymmetry, it would be effective in our experiment.

Comparing with NGA on small-world, we also use same experimental conditions to test SGA and NGA on regular lattices (the size of one-dimensional lattices is also 256 , and $1 \leq r_{n} \leq 128$ ).

\section{Results and Analysis}

\subsection{Experiment 1}

The results of experiment 1 are presented in Fig.2.1 and Fig.2.2. Two curves in the figure almost coincide, and success rate of NGA and SGA is $93 \%$ and $91 \%$ respectively. Therefore, we can imply NGA is "equivalent to" SGA, when it base on the fully connected networks. According to the results, we can ignore the influence of program in next experiments.

\subsection{The Benefits of Small-World}

The "success rate" is the percentage of successful runs. The success rates of different algorithms are presented in below table:

\begin{tabular}{|c|c|}
\hline Algorithm & Success Rate \\
\hline \hline NGA on small-world (Best) & 0.6750 \\
\hline NGA on small-world (Worst) & 0.4083 \\
\hline NGA on regular lattice (radius $=2)$ & 0.5750 \\
\hline NGA on regular lattice (Best, radius $=4$ ) & 0.6917 \\
\hline SGA & 0.3858 \\
\hline
\end{tabular}

From the table, we can find: 1) the worst performance of NGA on small-world is even better than the performance of SGA; 2) the best performance of NGA on small-world is much better than the performance of NGA on regular lattice on which small-world model base; 3 ) the best performances of NGA on regular lattice and on small-world are approximate sameness, but, comparing with regular lattice, average connected degrees reduce by half in small-world model, For 


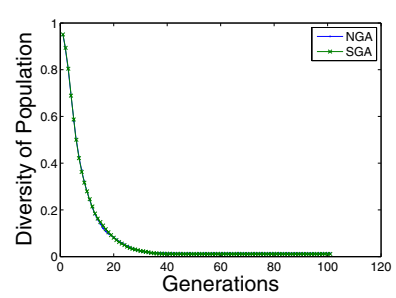

(1)

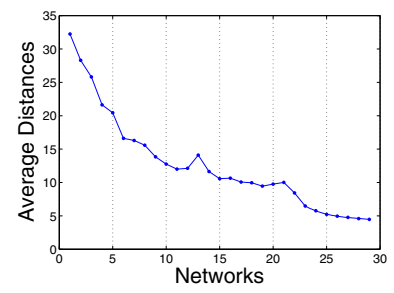

(3)

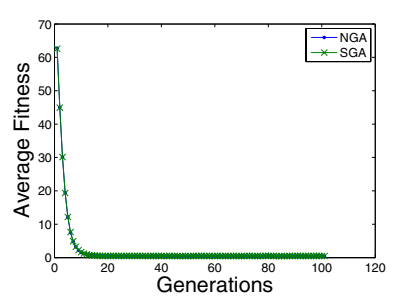

$(2)$

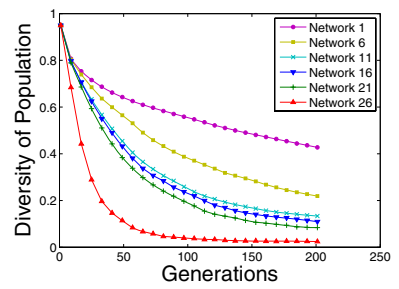

(4)

Fig. 2. Results of Experiment

(1) is graph of the average diversity of population at each generation. (2) is graph of average fitness of population at each generation (mirror the convergent speed of GA). (3) is graph of average distances for all 29 networks. (4) is graph of diversity of population at each generation for $G_{1}, G_{6}, G_{11}, G_{16}, G_{21}, G_{26}$.

parallel GA, it means that the cost of communication of network will decline dramatically.

In sum, the small-world plays a extraordinary role in promoting the performance of genetic algorithm.

\subsection{Relationships Between Average Vertex-to-Vertex Distances and the Diversity of Population}

The relationships between average vertex-to-vertex distances (labeled by $L$ ) and diversity is described by Fig.2.3 and Fig.2.4. There are two obvious factors: First, the changing rate of diversity increase with the decreasing of $L$; Second, $L$ of $G_{11}, G_{16}$ and $G_{21}$ is similar, at the same time, the diversity curves of $G_{11}, G_{16}$ and $G_{21}$ is also close to each other. Therefore, we can deduce that $L$ would determine the changing rate of diversity of population in spatial GA. It is reasonable. Shorter $L$ would promote individuals' diffusion. It let a few advantage segments of "gene" occupy more and more individuals in the population, and disadvantage segments of "gene" are replaced rapidly and disappear ultimately.

\subsection{Different Results from Previous Researches}

Jayshree Sarma and Kenneth De Jong's results [7 show that the critical parameter, which deeply affects the behavior of spatial GA, is the ratio (labeled by $R$ ) of 
radius of neighborhood to the radius of the underlying networks. However, from Fig.3, we find two opposite phenomenons: First, trends of two curves (Fig.3.2) aren't consistent totally; Second, the turning-points of two curves cannot match to each other. Although the improving from local selection is clear from above results, the two factors would support our opinion that $R$ cannot entirely determines the behavior of spatial GA on small-world, on the contrary, the crucial role of $R$ in evolution on regular lattices is obvious.

\subsection{New Discovery: The Influence of Asymmetric Topology}

From Fig.3.1, we can find an interesting phenomenon: two curves' turning-points are well matched to each other (these points include: $8,9,10,11,16,17,19,20$, 21), and there are just a few unmatched points, such as 15 and 22. All of those cannot be explained by contingency. Therefore, we could say asymmetric topology of underlying networks could also affects the behavior of spatial GA on small-world. I concede that two curves are unmatched at tail, however, I think the most possible reason is our asymmetric factor $U$ cannot correctly reflect the degree of asymmetry of small-world, when there are too many rewired links. In sum, the results of our experiments suggest that the influence of asymmetric topology cannot be ignored, when you design a spatial GA. Additionally, the performance of spatial GA might be further improved by well designed asymmetric topology.

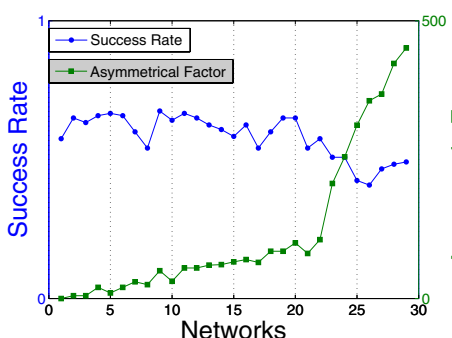

(1)

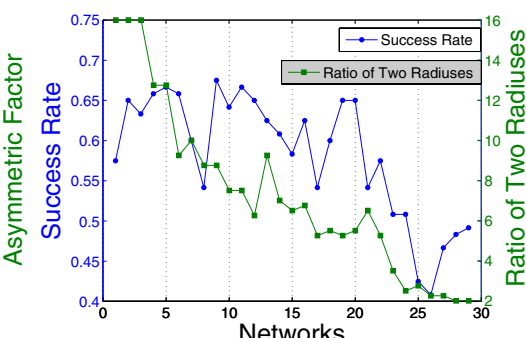

(2)

Fig. 3. Results of Experiment 2

(1) is the graph of asymmetric factor for all 29 networks; (2) is the graph of ratio of the average connected radius of the vertex to the radius of the underlying networks for all 29 networks.

\section{Conclusion and Future Works}

In our experiments, spatial GA on small-world exhibit its outstanding benefits, comparing with SGA and spatial GA on regular networks. In common sense, these benefits seem to be due to local selection effect. Furthermore, we have found that another factor, asymmetric topology of networks, also play a subtle role in 
the spatial GA, and we have given a few evidences to support this discovery. The discovery could provide us a potentially valuable tool to improve the performance of spatial GA, and also helps us to understand the behaviors of evolution on small-world.

Admittedly, our work is just a beginning of exploring spatial GA on smallworld networks. In the future, more works need to be done to analyze the properties of the behavior of spatial GA on small-world, such as more details about asymmetric topology, relationships between local selection and asymmetric topology on small-world, and how to develop more efficient genetic algorithm with asymmetric topology.

\section{References}

1. C. Hauert, M. Doebeli: Spatial structure often inhibits the evolution of cooperation in the snowdrift game. Nature, 428 (2004) 643-646

2. D. J. Watts, S. H. Strogatz: Collective dynamics of 'small-world' networks. Nature, 393 (1998) 440-442

3. E. Cantu-Paz: Efficient and Accurate Parallel Genetic Algorithm. Kluwer Academic Press (2000)

4. E. Lieberman, C. Hauert, M. A. Nowak: Evolutionary dynamics on graphs. Nauture, 433 (2005) 312-312

5. E. Alba, M. Tomassini: Parallelism and evolutionary algorithms. IEEE Transactions on Evolutionary Computation, 6(5) (2002) 443-462

6. H. Ebel, S. Bornholdt: Coevolutionary games on networks. Phys. Rev. E. 66 (2002) 56-118.

7. J. Sarma, K. De Jong: An analysis of the effects of neighborhood size and shape on local selection algorithms, Parallel Problem Solving from Nature IV, Berlin, (1996) 236-244

8. M.Nakamaru, H.Matsuda, Y.Iwasa: The evolution of cooperation in a latticestructured population.Journal of theoretical Biology, 184 (1997) 65-81

9. M. Giacobini, M. Tomassini, A. Tettamanzi: Takeover time curves in random and small-world structured populations. GECCO'05, June 25-29, 2005, Washington, D.C., USA

10. M. A. Nowak, R. M. May: The spatial dilemmas of evolution. Int. J. Bifurcation Chaos, 3 (1993) 35-78

11. N. Williams, M. Mitchell: Investigating the success of spatial coevolution. GECCO'05, June 25-29, 2005, Washington, D.C., USA

12. The Matlab's genetic algorithm and direct search toolbox documents: http://www.mathworks.com/access/helpdesk/help/toolbox/gads/ 